\title{
Introducing Pareto-based MOEA to Neurosurgery Preoperative Path Planning
}

\author{
Noura Hamze \\ ICube, Univ. de Strasbourg \\ 300 bd Sébastien Brant \\ 67412 Illkirch, France \\ IHU - 1 Place de l'Hôpital \\ 67000 Strasbourg, France \\ n.hamze@unistra.fr
}

\author{
Pierre Collet \\ ICube, Univ. de Strasbourg \\ 300 bd Sébastien Brant \\ 67412 Illkirch, France \\ collet@unistra.fr
}

\author{
Caroline Essert \\ ICube, Univ. de Strasbourg \\ 300 bd Sébastien Brant \\ 67412 Illkirch, France \\ IHU - 1 Place de l'Hôpital \\ 67000 Strasbourg, France \\ essert@unistra.fr
}

\begin{abstract}
This paper presents the first implementation of NSGA-II in neurosurgery preoperative path planning. Deep Brain Stimulation (DBS) is a surgical treatment of Parkinson's disease that can be regarded as a multi-objective optimization problem, searching for the best compromise between multiple electrode placement rules. Most of the current automatic decision-making processes use aggregative approaches with single objective optimization, even though they are known for their inability to find all Pareto-optimal solutions. Firstly, we show this is the case on 20 datasets of patients by comparing our implementation of NSGA-II to the weighted sum (WS) strategy. Then, we show that it requires about 9 hours to find equivalent results using a deterministic scan of the search space where NSGA-II does it in about 3mn. This paper presents an objective validation that even simple techniques such as NSGA-II should be used by surgeons over more intuitive weighted based methods.
\end{abstract}

\section{INTRODUCTION}

Planning a safe and efficient trajectory for a surgical tool is a crucial and a challenging task that requires a long experience. The path is usually chosen as the best compromise between multiple surgical rules that can often be contradictory. This is particularly difficult when the targeted structure is small and/or deeply located and the surrounding obstacles are numerous. DBS intervention is performed to decrease the tremors of Parkinson's disease. It consists in implanting a permanent electrode in a small deep nucleus of the brain, and is a good example of interventions for which such a preoperative trajectory planning is essential.

Several automatic path planning techniques have been proposed for DBS in the literature in the past years, for instance $[1,3,4]$. However, they are based on mono-objective optimization approaches: they combine all the surgical rules into a single aggregative cost function, after assigning impor-

Permission to make digital or hard copies of part or all of this work for personal or classroom use is granted without fee provided that copies are not made or distributed for profit or commercial advantage and that copies bear this notice and the full citation on the first page. Copyrights for third-party components of this work must be honored. For all other uses, contact the owner/author(s).

GECCO '16 July 20-24, 2016, Denver, CO, USA

(C) 2016 Copyright held by the owner/author(s).

ACM ISBN 978-1-4503-4323-7/16/07.

DOI: http://dx.doi.org/10.1145/2908961.2909028 tance weights to each objective, and minimize the obtained function to find an optimal planning strategy. This approach is intuitive, and sounds close to the current decision making process but is known to possibly lead to an under-detection of the optimal solutions in a given search space. The purpose of this work is therefore to better clarify and quantify the pros and cons of the different optimization approaches on the particular case of surgical trajectory planning and compare them to an exhaustive search on a discretization of the search space, in order to get a good comparison ground.

In this study, the three objectives to optimize for a DBS intervention are: the proximity to the standard trajectory defined by expert neurosurgeons and commonly used in commercial platforms, the distance from the electrode to the cortical sulci in which the vessels usually are located, and the distance from the electrode to the ventricles. These objectives apply to the anatomy of the patient's brain represented as a $3 \mathrm{D}$ model reconstructed from preoperative images.

\section{PROPOSED METHOD USING NSGA-II}

The proposed approach is based on a multi-objective evolutionary optimization method based on a Pareto ranking scheme: NSGA-II (Non-dominated Sorting Genetic Algorithm II) [2], with a complexity of $O\left(m N^{2}\right)$, where $m$ is the number of objectives and $N$ the population size.

The NSGA-II algorithm consists of two phases. Firstly, an initial population $\mathcal{P}_{0}$ of size $N$ is selected randomly over an initial solution space $\mathcal{S}$. All the individuals are evaluated using the three objective functions described above and ranked on $r$ Pareto fronts. Secondly, a generational evolutionary engine iteratively creates offspring populations $\mathcal{Q}_{n}$ of size $N$ by selecting parents using a $D B X$ Dominance Based Restricted selection on $\mathcal{P}_{n}$ based on the individuals' rank. Offsprings are created by crossover and mutation processes and all children are evaluated along the three different functions. Then, new ranks are established for population $\mathcal{P}_{n}+\mathcal{Q}_{n}$ as described above, and a new population $\mathcal{P}_{n+1}$ is formed by adding solutions from the first, second, third, ... fronts until the population size is reached. The solutions of the last accepted front are sorted according to a crowded distance calculated as described in [2].

The chosen crossover method (applied with probability of 0.9 and a crossover distribution index eta en $_{c}=10$ ) is BLX$\alpha$, where an offspring $c_{k}$ is generated by a random linear recombination of its parents $p_{1, k}$ and $p_{2, k}$ as follows: $c_{k}=$ $\left(1-\gamma_{i}\right) p_{1, k}+\gamma_{i} p_{2, k}$, where $\gamma_{i}=(1+2 \alpha) u_{i}-\alpha$ with $u_{i}$ 
a random number between 0 and 1 , and $\alpha=0.5$ in our experiments. Then a polynomial mutation function with a probability of 0.5 and a mutation distribution index eta $\mathrm{a}_{m}=5$ is applied. Child $c_{k}$ is mutated by: $c_{k}=c_{k}+\left(c_{k}^{u}-c_{k}^{l}\right) \delta_{k}$, where $c_{k}^{u}=\frac{c_{k}-b^{l}}{b^{u}-b^{l}}$ and $c_{k}^{l}=\frac{b^{u}-c_{k}}{b^{u}-b^{l}}$, with $b^{u}, b^{l}$ the upper and lower bounds of the solution space. $\delta_{k}$ is a small random variation calculated via a polynomial distribution.

Initialization is done with a population size of $N=2,000$ individuals randomly taken over the surface of feasible entry points over the skull (Fig.1a). The algorithm is run over 10 generations, and converges to an output front denoted $\mathcal{F}_{1}^{N S}$.

\section{REFERENCE METHODS}

\subsection{Weighted Sum}

In this method, the single cost function to minimize is expressed as a weighted sum of all individual objective functions with their respective weighting factors. A classical mono-criteria optimization technique is then used to minimize $f$ over the space of possible candidate trajectories. An initialization phase starts the optimization process close to a known approximated minimum is used to avoid premature convergence to a local optimum.

Usual approaches for DBS propose an interactive weight exploration, that allows the surgeon to change the weights and see possible alternative points. But even by changing the weights, points in concave Pareto fronts will not be found. To show this, we computed the set of "optimal" points obtained with WS by exploring automatically 20,000 possible weights combinations, thanks to a uniform Dirichlet distribution of the weights. Then we filtered out the sub-optimal points by computing its Pareto front $\mathcal{F}_{1}^{W S}$.

\subsection{Discretization Dominance-Based Method}

The third method (denoted DISC) is an exhaustive exploration of a dense uniform discretization of 20,000 points over the search space (Fig.1b). A Pareto front $\mathcal{F}_{1}^{D I}$ using the three objectives is calculated according to the strict dominance rule, and used as a ground truth for our experiments.

\section{RESULTS AND CONCLUSION}

Our retrospective study was performed on 3D brain models reconstructed from 20 preoperative Magnetic Resonance Images of 10 patients with Parkinson's disease who underwent bilateral DBS implant in the subthalamic nucleus. The tests were done on an Intel Core i7 2.67 GHz.

We compared the surfaces covered by the fronts generated by the different methods over the search space. Fig.1c illustrates in green the surfaces covered by $\mathcal{F}_{1}^{N S}$ and not dominated by points of $\mathcal{F}_{1}^{W S}$, and Fig.1d illustrates in red the surfaces covered by $\mathcal{F}_{1}^{N S}$ and not dominated by points of $\mathcal{F}_{1}^{D I}$, for one of the 20 datasets. The average areas for these surfaces are respectively $50 \mathrm{~mm}^{2}$ with a standard deviation equal to $41 \mathrm{~mm}^{2}$ and $148 \mathrm{~mm}^{2}$ with a standard deviation of $44 \mathrm{~mm}^{2}$, for an initial search space of $2,267 \mathrm{~mm}^{2}$ in average. Approximately $37 \%$ of the solutions found by NSGA-II could not be found by WS exploration. We also observe that with a similar number of samples, NSGA-II was always able to find many solutions missed by DISC. In order to estimate the level of discretization that would be required to obtain equivalent results with DISC we ran a search over

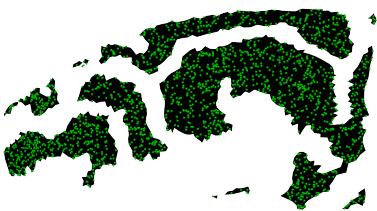

(a) NSGA-II: initial population (green)

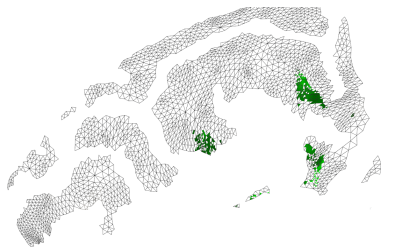

(c) Solutions found by NSGA-II not dominated by WS (green)

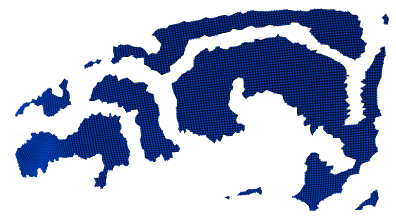

(b) DISC: initial discretization (blue)
Figure 1: Initial distributions and optimal areas found by NSGA-II and not by others, over the surface of feasible entry points (search space) on the left side of the skull

the number of samples. We found that to surpass NSGA-II, DISC would need around 650,000 points, with an average time required to compute the Pareto front of 8 hours 55 mins compared to around $3 \mathrm{~min}$ in average for NSGA-II.

This study highlighted that a Pareto-based multi-objective evolutionary algorithm (NSGA-II) is superior to two other approaches classically used in the framework of preoperative planning of DBS electrodes in neurosurgery. NSGA-II can find more optimal entry points than a weighted sum (even with a wide exploration of the weights) and than an exhaustive search over a discretization of the search space comparable to the literature (approx. 1 point every $\mathrm{mm}$ ). Finally, we showed that for a result comparable to NSGA-II, an exhaustive search would require a very dense discretization of the search space and a very high computation time that would not be compatible with clinical use.

\section{Acknowledgments}

This work was supported by the French National Research Agency under grant ANR 2010 BLAN 020902.

\section{REFERENCES}

[1] S. Bériault, F. A. Subaie, D. L. Collins, A. F. Sadikot, and G. B. Pike. A multi-modal approach to computer-assisted deep brain stimulation trajectory planning. Int J Comput Ass Rad, 7(5):687-704, 2012.

[2] K. Deb, A. Pratap, S. Agarwal, and T. Meyarivan. A fast and elitist multiobjective genetic algorithm: NSGA-II. IEEE T Evolut Comput, 6(2):182-197, 2002.

[3] C. Essert, C. Haegelen, F. Lalys, A. Abadie, and P. Jannin. Automatic computation of electrode trajectories for deep brain stimulation: a hybrid symbolic and numerical approach. Int J Comput Ass Rad, 7(4):517-532, 2012.

[4] R. R. Shamir, L. Joskowicz, I. Tamir, E. Dabool, L. Pertman, A. Ben-Ami, and Y. Shoshan. Reduced risk trajectory planning in image-guided keyhole neurosurgery. Med. Phys., 39(5):2885, 2012. 